J. DIFFERENTIAL GEOMETRY

56 (2000) 285-299

\title{
LAPLACIAN EIGENVALUES AND DISTANCES BETWEEN SUBSETS OF A MANIFOLD
}

\author{
JOEL FRIEDMAN \& JEAN-PIERRE TILLICH
}

\begin{abstract}
In this paper we give a new method to convert results dealing with graph theoretic (or Markov chain) Laplacians into results concerning Laplacians in analysis, such as on Riemannian manifolds. We illustrate this method by using the results of [6] to prove

$$
\lambda_{1} \leq \frac{1}{\operatorname{dist}^{2}(X, Y)}\left(\cosh ^{-1} \sqrt{\frac{\mu X^{\mathrm{c}} \mu Y^{\mathrm{c}}}{\mu X \mu Y}}\right)^{2} .
$$

for $\lambda_{1}$ the first positive Neumann eigenvalue on a connected compact Riemannian manifold, and $X, Y$ any two disjoint sets (and where $X^{\mathrm{c}}$ is the complement of $X$ ). This inequality has a version for the $k$-th positive eigenvalue (involving $k+1$ disjoint sets), and holds more generally for all "analytic" Laplacians described in [6]. We show that this inequality is optimal "to first order," in that it is impossible to obtain an inequality of this form with the right-hand-side divided by $1+\epsilon$ for any fixed constant $\epsilon>0$.
\end{abstract}

\section{Introduction}

This paper gives a new method for converting results on discrete math Laplacians, e.g., Laplacians on graphs or Markov chains, into results on analysis Laplacians, e.g., Laplacians on Riemannian manifolds. Converting in the reverse direction is found in [10], whose methods partially lead to the method in this paper. We shall illustrate our method on one class of examples, which we now describe.

To fix ideas, let $M$ be a compact connected Riemannian manifold, possibly with boundary, and $\lambda_{1}$ the smallest positive Neumann eigenvalue of the Laplacian. Then if $X, Y$ are disjoint Borel sets in $M$, Chung,

Received April 18, 2000. Research supported in part by an NSERC grant. 
Grigor'yan, and Yau, in [5], showed that

$$
\lambda_{1} \leq \frac{1}{\operatorname{dist}^{2}(X, Y)}\left(\log \frac{C(\mu M)^{2}}{\mu X \mu Y}\right)^{2}
$$

with $C=4$, where $\mu$ is the Riemannian volume measure (and where the logarithm is to base $e$ ). Later, in [6], they obtained inequality (1) with $C=e$, and Bobkov and Ledoux, in [2], obtained $C=1$. We mention that in [6] the above inequality holds for more general Riemannian manifolds and more general Laplacians, and in [2] holds in the even greater generality of probability metric spaces ${ }^{1}$. Also, we mention that such inequalities have appeared in the context of graphs and certain metric probability spaces, as in $[1,13,12,11,14,4,6]$ and the references there (specifically for graphs see [1] Theorem 2.6 with $a=1 / 2$, [12] Lemma 2.4 , and $[6])$.

In this paper we will prove the theorem below.

Theorem 1.1. Let $\mathcal{L}$ be an analytic Laplacian on a metric measure space $(M, \mu)$, and let $\lambda_{1}$ be the lower bound of the spectrum of $\mathcal{L}$ acting on the functions orthogonal to the constants. Then for $X, Y$ disjoint Borel subsets of $M$ we have

$$
\lambda_{1} \leq \frac{1}{\operatorname{dist}^{2}(X, Y)}\left(\cosh ^{-1} \sqrt{\frac{\mu X^{\mathrm{c}} \mu Y^{\mathrm{c}}}{\mu X \mu Y}}\right)^{2},
$$

where $X^{\mathrm{c}}$ is the complement of $X$.

This theorem will, in particular, apply to the second Neumann eigenvalue of a compact Riemannian manifold as above. In particular, using $\cosh ^{-1}(y) \leq \log (2 y)$ and that $\mu X^{\mathrm{c}}, \mu Y^{\mathrm{c}} \leq \mu M$, we conclude:

$$
\lambda_{1} \leq \frac{1 / 4}{\operatorname{dist}^{2}(X, Y)}\left(\log \frac{4(\mu M)^{2}}{\mu X \mu Y}\right)^{2} .
$$

Furthermore we give a simple class of examples to show that any inequality of the form

$$
\lambda_{1} \leq \frac{C_{1}}{\operatorname{dist}^{2}(X, Y)}\left(\log \frac{C_{2}(\mu M)^{2}}{\mu X \mu Y}\right)^{2}
$$

\footnotetext{
${ }^{1}$ For a metric probability space, $\lambda_{1}$ is defined as the optimal constant in a Poincaré inequality; no Laplacian need exist.
} 
for Neumann eigenvalues of compact Riemannian manifolds must have $C_{1} \geq 1 / 4$. So, if we take $\mu X$ and $\mu Y$ as "small" compared to $\mu M$, our inequality is optimal to first order. We also remark that we can fairly quickly see ${ }^{2}$ that the inequality in Theorem 1.1 is stronger $^{3}$ than that in Equation (1) with $C=1$ for all values of $\mu X$ and $\mu Y^{4}$.

Surprisingly, as indicated before, our result on Riemannian manifolds follows from our method of converting results from discrete math Laplacians to analysis Laplacians, along with the graph theoretic techniques of [6]. Our inequality will hold for any Riemannian manifold and Laplacian as mentioned there. We shall also give a version of Theorem 1.1 for higher eigenvalues.

In Section 2 we will explain our method of converting results on Laplacians in discrete math to those in analysis. In Section 3 we review the setup of [6] and say what we mean by an "analytic" Laplacian. In Section 4 we will use the method of Section 2 and the techniques in [6] to prove Theorem 1.1; we prove several variants of this theorem there, including one for $\lambda_{k}$ with $k>1$. In Section 5 we give a simple class of examples to show that we must have $C_{1} \geq 1 / 4$ in an inequality (3).

\section{The general method}

In this section we give a general method for converting results on discrete math Laplacians into those on analysis Laplacians. We will then describe how [10] partially indicates this approach.

Our idea has two steps. First, if $\Delta$ is negative semidefinite Laplacian in analysis, then $\widetilde{\Delta}=1-\cos \sqrt{-\Delta}$ has two properties of a graph theoretic Laplacian:

\footnotetext{
${ }^{2}$ Indeed, let $A=\mu M / \mu X$ and $B=\mu M / \mu Y$. It suffices to show that $\log (A B)$ exceeds $\cosh ^{-1} \sqrt{(A-1)(B-1)}$. Taking cosh of both sides reduces this to comparing $(A B) / 2+1 /(2 A B)$ to $\sqrt{(A-1)(B-1)}$. Squaring and multiplying by 4 reduces this to showing $(A B)^{2}+(A B)^{-2}+4 A+4 B-4 A B-2$ is positive. The gambit $4 A+4 B \geq 8 \sqrt{A B}$ implies that it suffices to show that $x^{2}+x^{-2}+8 \sqrt{x}-4 x-2$ is positive (for $x \geq 4$ ). A computer investigation shows that $y^{8}+1+8 y^{5}-4 y^{6}-2 y^{4}$ has no non-negative roots (where we substitute $y^{2}=x$ ); by hand one can also compute this polynomials Sturm sequence and apply the test between $y=0$ and $y=+\infty$ to see (by hand) that this polynomial has no positive roots.

3 However, the result of Bobkov and Ledoux holds for any metric probability space, so our result is stronger only when an analytic Laplacian exists.

4 Although Bobkov and Ledoux never mentioned it, it is possible to use the theorems in their paper to achieve any constant $C_{1}>1 / 4$, with a $C_{2}$ that depends on how close $C_{1}$ is to $1 / 4$, in Equation (3).
} 
(1) its spectrum ${ }^{5}$ lies in [0,2], and

(2) the support of $\widetilde{\Delta} u$ is within a distance of 1 to the support of $u$ for any function $u$ in $L^{2}$.

These properties, and possibly others, may make it possible to directly apply the same techniques used in discrete math to $\widetilde{\Delta}$.

The next step is to take the limit as the Riemannian metric is scaled ${ }^{6}$ by $1 / \epsilon$ and take $\epsilon \rightarrow 0$. This means that distances get scaled by $1 / \epsilon$. This also means, for example, that we work with $\epsilon^{2} \Delta$ instead of $\Delta$, and with $\widetilde{\Delta}$ being $1-\cos (\epsilon \sqrt{-\Delta})$.

Consider a situation where $-\Delta$ has eigenvalues $0=\lambda_{0}<\lambda_{1} \leq \lambda_{2} \leq$ $\cdots$. We have to be careful about what we say about the eigenvalues of $\widetilde{\Delta}^{\epsilon}=1-\cos (\epsilon \sqrt{-\Delta})$. While it does have eigenvalues $\nu_{i}=1-$ $\cos \left(\epsilon \sqrt{\lambda_{i}}\right)$ for each $i$, it is not true that $\nu_{1}$ is necessarily the second smallest eigenvalue. So if we want to use a technique involving the second smallest graph eigenvalue, we have to be careful to separate $\widetilde{\Delta}^{\epsilon}$ into its $\nu_{0}$ component, $\nu_{j}$ components for all $\nu_{j} \geq \nu_{1}$ and $j \geq 1$, and finally the $\nu_{j}<\nu_{1}$ and $j \geq 1$. This third component will, in some sense, go to zero as $\epsilon \rightarrow 0$, and if things workout we will have an interesting theorem involving $\nu_{0}$ and $\nu_{1} \approx \epsilon^{2} \lambda_{1} / 2$.

We now explain how we came to the first step in our method. With any graph one traditionally associates a positive semidefinite Laplacian, $\Delta_{G}$. In [10] another type of positive semidefinite Laplacian is introduced, a so-called "edge-based" Laplacian, $\Delta_{E}$, which much more closely resembles minus a Laplacian from analysis than does $\Delta_{G}$ (for example, the edge-based wave equation has wave propogation speed 1 , where as that based on $\Delta_{G}$ has infinite speed). There are senses in which we may view $\Delta_{G}$ as similar to $1-\cos \sqrt{\Delta_{E}}$. This suggests that if $\Delta$ is a Laplacian from analysis, by forming $\widetilde{\Delta}=1-\cos \sqrt{-\Delta}$ we get a Laplacian that much more closely resembles a graph theoretic Laplacian.

\section{Analytic Laplacians}

In this section we explain what is meant by an "analytic Laplacian." We begin by reviewing the setup in [6]. We assume $M$ is a metric

\footnotetext{
${ }^{5}$ Graph theoretic and Markov chain Laplacians have their spectrum lying in $[0,2]$ if the Laplacians are appropriately normalized, as in $[8,9,7,6,10]$.

${ }^{6}$ By scaling the metric by $c$ we mean that in $g=g_{i j} d x^{i} d x^{j}$ we multiply the $g_{i j}$ by $c^{2}$.
} 
space with Borel measure $\mu$ with $\mu M<\infty$. Let $\mathcal{L}$ be an operator on $L^{2}(M, \mu)$ (possibly unbounded) with for each $s \in[0, \infty)$ a function $P_{s}: \operatorname{spec}(\mathcal{L}) \rightarrow \mathbb{R}$ with:

1. $\mathcal{L}$ is non-negative and self-adjoint.

2. $\operatorname{Dom}(\mathcal{L})$ is dense in $L^{2}(M, \mu)$ and contains the constants, and $\mathcal{L}$ maps the constants to 0 .

3. $P_{s}(\mathcal{L})$ is bounded for each $s$ with

$$
\operatorname{supp}\left(P_{s}(\mathcal{L}) u\right) \subset\{x \in M \mid \operatorname{dist}(x, \operatorname{supp} u) \leq s\}
$$

for each $u \in L^{2}(M, \mu)$, i.e., $P_{s}(\mathcal{L})$ enlarges the support ${ }^{7}$ of any function by a distance of at most $s$.

Definition 3.1. If the above conditions hold, we say that $\mathcal{L}$ is a standard Laplacian with propagation function $P_{s}$. We say that $\mathcal{L}$ is analytic if the above holds with $P_{s}(\lambda)=\cos (s \sqrt{\lambda})$.

Many examples are given in [6] of such $M, \mu, \mathcal{L}$ with $\mathcal{L}$ an analytic Laplacian; they include:

1. $M$ is a complete Riemannian manifold (without boundary) of finite volume. $\mathcal{L}=-\Delta$ with $\Delta$ the Laplace operator; more precisely, $\mathcal{L}$ is the unique self-adjoint extension of $-\Delta$ acting on $C_{\mathrm{c}}^{\infty}(M)$.

2. $M$ is a compact Riemannian manifold with boundary or a compact region of some other Riemannian manifold. $\mathcal{L}$ is as in item 1 , imposing the Neumann boundary condition.

3. $M$ is a complete Riemannian manifold, possibly of infinite volume. $\sigma \in L^{1}\left(M, \mu_{0}\right)$ is smooth, where $\mu_{0}$ is the Riemannian volume, such that with respect to $\mu=\sigma \mu_{0}, M$ is of finite volume. $\mathcal{L}$ is formed from $-\sigma^{-1} \nabla \cdot(\sigma \nabla)$.

In [6] it is remarked that if the greatest lower bound of the spectrum of $\mathcal{L}$ is $\lambda_{0}$, rather than 0 , and $\lambda_{0}$ has a corresponding positive eigenfunction, $\psi$, then $\psi^{-1} \circ \mathcal{L} \circ \psi-\lambda_{0}$ will be analytic. So a variant of theorems on analytic Laplacians hold for such Laplacians.

\footnotetext{
${ }^{7}$ By the support of a function, $u$, in $L^{2}$ we mean the support in the distribution sense, i.e., the complement of the union of all open sets $\Omega$ such that $\int \phi u=0$ for, say, all bounded, continuous $\phi$ supported in $\Omega$.
} 


\section{A proof of Theorem 1.1}

We now assume that $\mathcal{L}$ is analytic, and set $E$ to be the associated spectral measure, mapping Borel sets on $\mathbb{R}$ to projections on $L^{2}(M, \mu)$. Let us assume that Spec $\mathcal{L} \subset\{0\} \cup\left[\lambda_{1}, \infty\right)$ for some $\lambda_{1}>0$, and that $E(\{0\})$ is the projection onto the constants. If $E(\{0\})$ is not the projection onto the constants, then $\lambda_{1}=0$ and there is nothing to prove. Set

$$
I=\left\{\lambda \in \operatorname{spec} \mathcal{L} \mid \cos \sqrt{\lambda}>\cos \sqrt{\lambda_{1}}\right\}
$$

and let

$$
\pi_{0}=E(\{0\}), \quad \pi_{-1}=E(I \backslash\{0\}), \quad \pi_{1}=E(\operatorname{spec} \mathcal{L} \backslash I) .
$$

$\Delta_{G}$ defined to be $1-\cos \sqrt{\mathcal{L}}$ has properties similar to a graph theory Laplacian, in that $\Delta_{G}$ is symmetric and positive semidefinite, $\Delta_{G}$ maps the constants to zero, $\Delta_{G}$ is bounded (namely $\left\|\Delta_{G}\right\| \leq 2$ ), and finally $\Delta_{G}$ enlarges supports of functions by at most a distance of 1 . If also follows that if $Q_{d}$ is any polynomial of degree $\leq d$, then $Q_{d}\left(\Delta_{G}\right)$ expands supports by at most a distance of $d$. Now, if $u, v \in L^{2}(M, \mu)$ have supports whose distance between them is $>d$, then

$$
\left(Q_{d}\left(\Delta_{G}\right) u, v\right)=0
$$

Let $A_{i}=Q_{d}\left(\Delta_{G}\right) \circ \pi_{i}$ for $i \in\{-1,0,1\}$, and note that

$$
\left(Q_{d}\left(\Delta_{G}\right) u, v\right)=\left(A_{0} u, \pi_{0} v\right)+\left(A_{1} u, \pi_{1} v\right)+\left(A_{-1} u, \pi_{-1} v\right) .
$$

Since

$$
\begin{gathered}
\left|\left(A_{0} u, \pi_{0} v\right)\right|=\left|Q_{d}(0)\right|\left\|\pi_{0} u\right\|_{2}\left\|\pi_{0} v\right\|_{2}, \\
\left|\left(A_{1} u, \pi_{1} v\right)\right| \leq\left\|\pi_{1} u\right\|_{2}\left\|\pi_{1} v\right\|_{2} \sup _{\lambda \in \operatorname{spec} \mathcal{L} \backslash I}\left|Q_{d}(1-\cos \sqrt{\lambda})\right|, \\
\left|\left(A_{-1} u, \pi_{-1} v\right)\right| \leq\left\|\pi_{-1} u\right\|_{2}\left\|\pi_{-1} v\right\|_{2} \sup _{\lambda \in I \backslash\{0\}}\left|Q_{d}(1-\cos \sqrt{\lambda})\right|,
\end{gathered}
$$

we obtain

$$
\begin{aligned}
\left\|\pi_{1} u\right\|_{2}\left\|\pi_{1} v\right\|_{2}\left[\sup _{\lambda \in \operatorname{spec} \mathcal{L} \backslash I}\left|Q_{d}(1-\cos \sqrt{\lambda})\right|\right] \\
\geq\left\|\pi_{0} u\right\|_{2}\left\|\pi_{0} v\right\|_{2}\left|Q_{d}(0)\right| \\
\quad-\left\|\pi_{-1} u\right\|_{2}\left\|\pi_{-1} v\right\|_{2}\left[\sup _{\lambda \in I}\left|Q_{d}(1-\cos \sqrt{\lambda})\right|\right]
\end{aligned}
$$


Note that this inequality is just a variant of Proposition 2.1 in $[6](\bar{u}$ and $u^{(1)}$ in [6] correspond respectively to our $\pi_{0} u$ and $\pi_{1} u+\pi_{-1} u$ ). Now set $T_{n}$ to be the standard Chebychev polynomial determined via

$$
T_{n}(\cos \alpha)=\cos (n \alpha),
$$

(we may replace cos by cosh if we like), and set ${ }^{8}$

$$
Q_{d}(x)=T_{d}(\ell(x)) \quad \text { where } \quad \ell(x)=\frac{2+\lambda_{G, 1}-2 x}{2-\lambda_{G, 1}}
$$

where we set $\lambda_{G, 1}=1-\cos \sqrt{\lambda_{1}}$ and we assume that $d$ is an integer (compare this choice to the proof of Theorem 4.3 in [6]). Since $\ell\left(\lambda_{G, 1}\right)=$ 1 and $\ell(2)=-1, Q_{d}$ maps $\left[\lambda_{G, 1}, 2\right]$ to $[-1,1]$. Also

$$
\sup _{\lambda \in I}\left|Q_{d}(1-\cos \sqrt{\lambda})\right| \leq Q_{d}(0)=T_{d}\left(\frac{2+\lambda_{G, 1}}{2-\lambda_{G, 1}}\right) .
$$

Hence we have

$$
\begin{aligned}
\left\|\pi_{1} u\right\|_{2}\left\|\pi_{1} v\right\|_{2} \geq & {\left[\left\|\pi_{0} u\right\|_{2}\left\|\pi_{0} v\right\|_{2}\right.} \\
& \left.-\left\|\pi_{-1} u\right\|_{2}\left\|\pi_{-1} v\right\|_{2}\right] T_{d}\left(\frac{2+\lambda_{G, 1}}{2-\lambda_{G, 1}}\right) .
\end{aligned}
$$

Now let us fix $u, v \in L^{2}(M, \mu)$ and apply the same argument with $\Delta_{G}^{\epsilon}=1-\cos (\epsilon \sqrt{\mathcal{L}})$ for small $\epsilon>0$; now $\Delta_{G}^{\epsilon}$ extends supports by at most a distance $\epsilon$. We conclude that we may use $T_{d / \epsilon}$ instead of $T_{d}$ in the above argument and that we have ${ }^{9}$

$$
\left\|\pi_{1}^{\epsilon} u\right\|_{2}\left\|\pi_{1}^{\epsilon} v\right\|_{2} \geq\left[\left\|\pi_{0} u\right\|_{2}\left\|\pi_{0} v\right\|_{2}-\left\|\pi_{-1}^{\epsilon} u\right\|_{2}\left\|\pi_{-1}^{\epsilon} v\right\|_{2}\right] T_{d / \epsilon}\left(\frac{2+\lambda_{G, 1}^{\epsilon}}{2-\lambda_{G, 1}^{\epsilon}}\right),
$$

where $\pi_{ \pm 1}^{\epsilon}$ is as before, based on

$$
I^{\epsilon}=\left\{\lambda \in \operatorname{spec} \mathcal{L} \mid \cos (\epsilon \sqrt{\lambda})>\cos \left(\epsilon \sqrt{\lambda_{G, 1}}\right)\right\}
$$

(and we are assuming that $d / \epsilon \in \mathbb{Z}$ ). Clearly

$$
I^{\epsilon} \backslash\{0\} \subset(\operatorname{spec} \mathcal{L}) \cap\left[\left(2 \pi-\epsilon \sqrt{\lambda_{G, 1}}\right)^{2} / \epsilon^{2}, \infty\right),
$$

\footnotetext{
${ }^{8}$ The point of choosing $Q_{d}$ like this is that the maximum over all polynomials $P$ of degree $d$ of $\frac{P(0)}{\max _{x \in\left[\lambda_{G, 1}, 2\right]}|P(x)|}$ is attained for $P=Q_{d}$.

${ }^{9}$ Another way to view this approach is to think of the case in Riemannian geometry, and to scale the metric by $1 / \epsilon$ (i.e., in $g=g_{i j} d x^{i} d x^{j}$ we scale the $g_{i j}$ by $1 / \epsilon^{2}$ ). This scales distances by $1 / \epsilon$, scales the Laplacian by $\epsilon^{2}$, etc.
} 
and so $\pi_{-1}^{\epsilon} u \rightarrow 0$ and similarly for $v$ as $\epsilon \rightarrow 0^{+}$. Hence as $\epsilon \rightarrow 0^{+}$we also get $\pi_{1}^{\epsilon} u \rightarrow u-\pi_{0} u$ and similarly for $v$. We also have that as $\epsilon \rightarrow 0^{+}$

$$
\begin{aligned}
\lambda_{G, 1}^{\epsilon} & =1-\cos \left(\epsilon \sqrt{\lambda_{1}}\right)=\epsilon^{2} \lambda_{1} / 2+O\left(\epsilon^{4}\right) \\
\frac{2+\lambda_{G, 1}^{\epsilon}}{2-\lambda_{G, 1}^{\epsilon}} & =1+\epsilon^{2} \lambda_{1} / 2+O\left(\epsilon^{4}\right) \\
\cosh ^{-1}\left(\frac{2+\lambda_{G, 1}^{\epsilon}}{2-\lambda_{G, 1}^{\epsilon}}\right) & =\epsilon \sqrt{\lambda_{1}}+O\left(\epsilon^{2}\right) \\
T_{d / \epsilon}\left(\frac{2+\lambda_{G, 1}^{\epsilon}}{2-\lambda_{G, 1}^{\epsilon}}\right) & =\cosh \left(d \sqrt{\lambda_{1}}\right)+O(\epsilon) .
\end{aligned}
$$

So taking $\epsilon \rightarrow 0^{+}$with $d / \epsilon \in \mathbb{Z}$, and then taking $d$ tending to the distance between the supports of $u$ and $v$, we conclude the following.

Theorem 4.1. Let $M, \mu, \mathcal{L}, \lambda_{1}$ be as before with $\mathcal{L}$ analysis-like. Then for any $u, v \in L^{2}(M, \mu)$ whose supports are at a distance $d$, we have

$$
\cosh \left(d \sqrt{\lambda_{1}}\right) \leq \frac{\left\|u-\pi_{0} u\right\|_{2}\left\|v-\pi_{0} v\right\|_{2}}{\left\|\pi_{0} u\right\|_{2}\left\|\pi_{0} v\right\|_{2}}
$$

Taking $u$ and $v$ to be the characteristic functions of two disjoint sets, $X, Y$, we conclude

$$
d \sqrt{\lambda_{1}} \leq \cosh ^{-1} \sqrt{\frac{\mu X^{\mathrm{c}} \mu Y^{\mathrm{c}}}{\mu X \mu Y}},
$$

where $d=\operatorname{dist}(X, Y)$. This proves Theorem 1.1. $\quad$ q.e.d.

The following theorem mildly generalizes the case where $\mathcal{L}$ is analytic; we do not know if it has any interesting applications; its proof is the same as the above.

Theorem 4.2. Let $M, \mu, \lambda_{1}, \mathcal{L}$ be as above, with $\mathcal{L}$ a standard Laplacian. Let $P_{s}$ be a propagation function such that:

(1) There exist constants $C, \alpha$ such that

$$
P_{\epsilon}(\lambda)=C \epsilon^{2} \lambda^{\alpha}+O\left(\epsilon^{3}\right)
$$

for any $\lambda$. 
$(2)$

$$
P_{\epsilon}(\operatorname{spec} \mathcal{L}) \subset\left[0, B_{\epsilon}\right]
$$

with

$$
\liminf _{\epsilon \rightarrow 0} B_{\epsilon}=B>0 .
$$

Then the preceeding theorem and corollary hold with $\sqrt{\lambda_{1}}$ replaced by $\left(4 C \lambda_{1}^{\alpha} / B\right)^{1 / 2}$.

As a silly example of this theorem, we may take $\mathcal{L}=(-\Delta)^{k}$ where $\Delta$ is a Riemannian Laplacian and $k$ a positive integer, and set $P_{s}(\lambda)=$ $1-\cos \left[s \lambda^{1 / k}\right]$ (and thereby recover the $k=1$ result).

Finally we remark that the generalization of $[6]$ to the $(k+1)$-st eigenvalue, $\lambda_{k}(\mathcal{L})$, of $\mathcal{L}$, holds as well. More precisely, let $k \geq 1$ be an integer, and let $\lambda_{k}$ be such that $E\left(\left[0, \lambda_{k}\right)\right)$ is a projection onto a subspace of dimension $k$.

Theorem 4.3. Let $u_{0}, \ldots, u_{k}$ be $k+1$ functions in $L^{2}(M, \mu)$ with supports which are at distance $\geq d$ of each other. Then

$$
\cosh \left(d \sqrt{\lambda_{k}}\right) \leq \max _{i \neq j} \frac{\left\|u_{i}-\pi_{0} u_{i}\right\|_{2}\left\|u_{j}-\pi_{0} u_{j}\right\|_{2}}{\left\|\pi_{0} u_{i}\right\|_{2}\left\|\pi_{0} u_{j}\right\|_{2}} .
$$

Proof. We simply add the ideas of the proof of Proposition 2.2 in [6] into what we have already done. First of all, we may assume that $\left(\pi_{0} u_{i}, \pi_{0} u_{j}\right) \geq 0$ for each $i, j$, by having $\pi_{0} u_{i}$ be a non-negative constant for each $i$, replacing $u_{i}$ by $-u_{i}$ if necessary.

Let $I$ and $Q_{d}$ be as before, with $\lambda_{k}$ replacing $\lambda_{1}$, and set

$$
\tilde{\pi}_{0}=E\left(\left[0, \lambda_{k}\right)\right), \quad \pi_{-1}=E\left(I \backslash\left[0, \lambda_{k}\right)\right), \quad \pi_{1}=E(\operatorname{spec} \mathcal{L} \backslash I) .
$$

Since the supports of $u_{i}$ and $u_{j}$ are at distance $>d$ for $i \neq j$ we obtain

$$
\left(Q_{d}\left(\Delta_{G}\right) u_{i}, u_{j}\right)=0
$$

and therefore

$$
-\left(Q_{d}\left(\Delta_{G}\right) \pi_{1} u_{i}, \pi_{1} u_{j}\right)=\left(Q_{d}\left(\Delta_{G}\right) \widetilde{\pi}_{0} u_{i}, \tilde{\pi}_{0} u_{j}\right)+\left(Q_{d}\left(\Delta_{G}\right) \pi_{-1} u_{i}, \pi_{-1} u_{j}\right) .
$$

Hence

$$
\begin{aligned}
\left\|\pi_{1} u\right\|_{2}\left\|\pi_{1} v\right\|_{2} \geq & \left|\left(Q_{d}\left(\Delta_{G}\right) \pi_{1} u_{i}, \pi_{1} u_{j}\right)\right| \\
\geq & \left|\left(Q_{d}\left(\Delta_{G}\right) \widetilde{\pi}_{0} u_{i}, \widetilde{\pi}_{0} u_{j}\right)\right|-\left|\left(Q_{d}\left(\Delta_{G}\right) \pi_{-1} u_{i}, \pi_{-1} u_{j}\right)\right| \\
\geq & \left|\left(Q_{d}\left(\Delta_{G}\right) \widetilde{\pi}_{0} u_{i}, \widetilde{\pi}_{0} u_{j}\right)\right| \\
& \quad-\sup _{\lambda \in I}\left|Q_{d}(1-\cos \sqrt{\lambda})\right|\left\|\pi_{-1} u_{i}\right\|_{2}\left\|\pi_{-1} u_{j}\right\|_{2} .
\end{aligned}
$$


Let $\pi_{0}$ be the projection onto the constants, and set

$$
\pi_{\text {new }}=\tilde{\pi}_{0}-\pi_{0}
$$

Then $\pi_{\text {new }}$ is a projection onto a subspace of dimension $k-1$, and defining

$$
\langle u, v\rangle=\left(Q_{d}\left(\Delta_{G}\right) \pi_{\text {new }} u, \pi_{\text {new }} v\right)
$$

gives a positive definite inner product, provided that $Q_{d}$ maps $\left[0, \lambda_{G, k}\right)$ to the positive reals. Our choice of $Q_{d}$ (with $\lambda_{G, k}=1-\cos \sqrt{\lambda_{k}}$ replacing $\left.\lambda_{G, 1}\right)$ clearly does so.

Since for any $k+1$ vectors in a $k-1$ dimensional Euclidean space there are two with a non-negative inner product (see [5]), there are $i, j$ with $\left\langle u_{i}, u_{j}\right\rangle \geq 0$. For this $i, j$ we have

$$
\begin{aligned}
\left(Q_{d}\left(\Delta_{G}\right) \widetilde{\pi}_{0}\left(u_{i}\right), \widetilde{\pi}_{0}\left(u_{j}\right)\right) & =\left(Q_{d}(0) \pi_{0}\left(u_{i}\right), \pi_{0}\left(u_{j}\right)\right)+\left\langle u_{i}, u_{j}\right\rangle \\
& \geq\left(Q_{d}(0) \pi_{0}\left(u_{i}\right), \pi_{0}\left(u_{j}\right)\right)
\end{aligned}
$$

For this $i, j$ we have:

$\left\|\pi_{1} u_{i}\right\|_{2}\left\|\pi_{1} u_{j}\right\|_{2} \geq\left[\left\|\pi_{0} u_{i}\right\|_{2}\left\|\pi_{0} u_{j}\right\|_{2}-\left\|\pi_{-1} u_{i}\right\|_{2}\left\|\pi_{-1} u_{j}\right\|_{2}\right] T_{d}\left(\frac{2+\lambda_{G, k}}{2-\lambda_{G, k}}\right)$.

Repeating this argument with $\Delta_{G}^{\epsilon}=1-\cos (\epsilon \sqrt{\mathcal{L}})$ as before yields the theorem.

Corollary 4.4. Let $X_{0}, \ldots, X_{k}$ be $k+1$ disjoint Borel subsets of $M$, whose distance between any two is at least $d$, and with other notation as before. Then

$$
\lambda_{k} \leq \frac{1}{d^{2}} \max _{i \neq j}\left(\cosh ^{-1} \sqrt{\frac{\mu X_{i}^{\mathrm{c}} \mu X_{j}^{\mathrm{c}}}{\mu X_{i} \mu X_{j}}}\right)^{2} .
$$

\section{An example extremal to first order}

In this section we give a simple and more or less well-known example to show that the optimal constant, $C_{1}$, in the inequality (3) is indeed $1 / 4$, as long as the inequality holds for the Neumann eigenvalues of manifolds with boundaries. If the inequality also holds for metric probability spaces, then $C_{1} \geq 1 / 4$ can be easily seen from the standard "exponential distribution" (studied in, for example, [2]); this space is 
just the non-negative reals with the usual metric, endowed with the probability measure $e^{-x} d x$. To emulate this in a Riemannian manifold, we take the surface of revolution generated by $y=e^{-x}$ for $x$ in an interval, $[L, R]$, with $L$ and $R-L$ large $^{10}$; these and related surfaces are well known examples of interesting spectral phenomena - see [3] for example $^{11}$. We will see that in our case we must take $L \rightarrow \infty$ with $(R-L) \rightarrow \infty$ to conclude that $C_{1} \geq 1 / 4$.

So we consider for reals $L<R$ the surface of revolution of $y=e^{-x}$ for $x$ between $L$ and $R$,

$$
M_{L, R}=\left\{(x, y, z) \in \mathbb{R}^{3} \mid y^{2}+z^{2}=e^{-2 x}, x \in[L, R]\right\} .
$$

We shall prove the following.

Lemma 5.1. For $\lambda_{1}$ being first non-zero Neumann eigenvalue of $M_{L, R}$ we have

$$
\lambda_{1}\left(M_{L, R}\right) \geq \frac{1}{4\left(1+e^{-2 L}\right)} .
$$

Lemma 5.2. Let $X, Y$ be the subsets of $M_{L, R}$ consisting of those points $(x, y, z)$ with $x \in[L, L+1]$ and, respectively, $x \in[R-1, R]$. Then $\operatorname{dist}(X, Y) \geq R-L-2$ and

$$
\log \frac{(\mu M)^{2}}{\mu X \mu Y} \leq R-L+1-2 \log (e-1)+\log \left(1+e^{-2 L}\right) .
$$

An immediate consequence of these two lemmas is the following theorem.

Theorem 5.3. Let Equation (3) hold for all compact manifolds with boundary with the Neumann condition. Then $C_{1} \geq 1 / 4$.

Since $C_{2}$ can never be less than $1 / 4$ (or else take $\mu X=\mu Y$ to be nearly $(\mu M) / 2$ ), our lemmas give no improvement over this trivial bound on $C_{2}$ for the case $C_{1}=1 / 4$.

We remark that if Equation (3) holds only for compact manifolds without boundary, then it seems probable to the authors that one can add "caps" or "bulbs" (as in [3]) to the $x=L$ and $x=R$ ends and get a similar theorem.

\footnotetext{
${ }^{10}$ Richard Froese has pointed out that we could simplify this section by modifying our Riemannian metric. Namely, we could take $d s^{2}=d t^{2}+e^{-2 t} d \theta^{2}$ for the metric (see below), and then all the $1+e^{-2 t}$ and $1+e^{-2 L}$ factors would become 1 .

${ }^{11}$ We remark that [3] is mentioned in [6] as evidence that their inequality might be improvable by roughly a factor of $1 / 4$.
} 
To prove the lemmas we recall some standard formulas in Riemannian geometry. First, if a Riemannian manifold has local coordiates $x^{1}, \ldots, x^{n}$ and Riemannian metric $g=g_{i j} d x^{i} d x^{j}$, then recall (using the standard summation convention and the convention that $a_{, j}$ denotes $\left.\partial a / \partial x^{j}\right)$

$$
\begin{aligned}
d V_{g} & =\sqrt{\operatorname{det} g} d x^{1} \cdots d x^{n} \\
\nabla u & =g^{i j} u_{, j}\left(\partial / \partial x^{i}\right) \\
|\nabla u|^{2} & =g^{i j} u_{, i} u_{, j} \\
\Delta u & =(\operatorname{det} g)^{-1 / 2}\left(\sqrt{\operatorname{det} g} g^{i j} u_{, i}\right)_{, j}
\end{aligned}
$$

where $d V_{g}$ is the Riemannian volume and $u$ a sufficiently smooth function. Placing coordinates $t \in[L, R]$ and $\theta \in \mathbb{R} /(2 \pi \mathbb{Z})$ on $M_{L, R}$ via

$$
x=t, \quad y=e^{-t} \cos \theta, \quad z=e^{-t} \sin \theta,
$$

the metric induced from $\mathbb{R}^{3}$ to $M_{L, R}$ is

$$
d s^{2}=d x^{2}+d y^{2}+d z^{2}=\left(1+e^{-2 t}\right) d t^{2}+e^{-2 t} d \theta^{2} .
$$

Plugging in $g_{11}=1+e^{-2 t}, g_{12}=g_{21}=0$, and $g_{22}=e^{-2 t}$ into the above formulas yields:

$$
\begin{aligned}
d V_{g} & =e^{-t} \sqrt{1+e^{-2 t}} d t d \theta \\
|\nabla u|^{2} & =\left(1+e^{-2 t}\right)^{-1} u_{t}^{2}+e^{2 t} u_{\theta}^{2} \\
\Delta u & =\frac{1}{e^{-t} \sqrt{1+e^{-2 t}}}\left[\left(\frac{e^{-t} u_{t}}{\sqrt{1+e^{-2 t}}}\right)_{t}+\left(\frac{\sqrt{1+e^{-2 t}}}{e^{-t}}\right) u_{\theta \theta}\right] .
\end{aligned}
$$

We finish by proving the lemmas. The first statement of Lemma 5.2 just follows from the fact that $X$ and $Y$ are separated by $R-L-2$ in their $x$ coordinate alone. For the second statement, we have

$$
\mu X=\int_{L}^{L+1} 2 \pi e^{-t} \sqrt{1+e^{-2 t}} d t \geq \int_{L}^{L+1} 2 \pi e^{-t} d t=2 \pi e^{-L-1}(e-1)
$$

and similary for $\mu Y$. Combining this with

$$
\mu M=\int_{L}^{R} 2 \pi e^{-t} \sqrt{1+e^{-2 t}} d t \leq 2 \pi \sqrt{1+e^{-2 L}} \int_{L}^{\infty} e^{-t} d t
$$

yields the lemma. 
Lemma 5.1 requires more computation. Let $f$ be the eigenfunction corresponding to $\lambda_{1}$. We shall prove Lemma 5.1 by first assuming that $f=f(t)$ is independent of $\theta$. Then we will show that this assumption is justified.

So assume $f=f(t)$. Then $f$, as a function of $t$ alone, is the second Neumann eigenfunction for the Rayleigh quotient

$$
\begin{aligned}
\mathcal{R}(u) & =\frac{\int|\nabla u|^{2} d V_{g}}{\int u^{2} d V_{g}} \\
& =\frac{\int_{L}^{R} u_{t}^{2}\left(e^{-t} / \sqrt{1+e^{-2 t}}\right) d t}{\int_{L}^{R} u^{2} e^{-t} \sqrt{1+e^{-2 t}} d t} .
\end{aligned}
$$

Hence

$$
\mathcal{R}(u) \geq\left(1+e^{-2 L}\right)^{-1} \widetilde{\mathcal{R}}(u),
$$

where

$$
\widetilde{\mathcal{R}}(u)=\frac{\int_{L}^{R} u_{t}^{2} e^{-t} d t}{\int_{L}^{R} u^{2} e^{-t} d t} .
$$

It follows from the min-max principle that $\lambda_{1}$ is at least as big as $\left(1+e^{-2 L}\right)^{-1}$ times the second Neumann eigenvalue for $\widetilde{\mathcal{R}}$; this later eigenvalue, $\lambda$, has corresponding eigenfunction $u$ with

$$
\left(e^{-t} u_{t}\right)_{t}=-\lambda u e^{-t}
$$

i.e.,

$$
u_{t t}-u_{t}+\lambda u=0 .
$$

The general solution to the last equation is

$$
u(t)=A_{1} e^{r_{1} t}+A_{2} e^{r_{2} t}, \quad \text { where } \quad r_{1,2}=\frac{1 \pm \sqrt{1-4 \lambda}}{2}
$$

provided that $\lambda \neq 1 / 4$. The Neumann conditions at $t=L$ and $t=R$ are immediately seen to imply that $(R-L)\left(r_{2}-r_{1}\right) \in 2 \pi i \mathbb{Z}$, and in particular $\lambda>1 / 4$. Hence either $\lambda=1 / 4$ or $\lambda>1 / 4$ for the second Neumann eigenvalue. It remains to show that $f=f(t)$.

To show that $f=f(t)$, assume to the contrary that $f$ depends on $\theta$. We will use separation of variables to show that $\lambda_{1}$ would then be larger than $1 / 4$ (assuming, say, $L \geq 0$ ). Indeed, solutions to $\Delta u=-\lambda u$ satisfy

$$
\left(h_{1} u_{t}\right)_{t}+h_{2} u_{\theta \theta}=-\lambda h_{3} u,
$$


where the $h_{i}=h_{i}(t)$ are given by

$$
h_{1}=\frac{e^{-t}}{\sqrt{1+e^{-2 t}}}, \quad h_{2}=\frac{\sqrt{1+e^{-2 t}}}{e^{-t}}, \quad \text { and } \quad h_{3}=e^{-t} \sqrt{1+e^{-2 t}} .
$$

If $u$ has the form $A(t) B(\theta)$, then we have

$$
B^{\prime \prime} / B=\left[-\lambda h_{3}-\left(h_{1} A^{\prime}\right)^{\prime} / A\right] / h_{2}
$$

which must be constant, since the left- and right-hand-sides respectively depend only on $t$ and $\theta$, respectively. Hence we can take $B(\theta)=e^{i n \theta}$ with $n \in \mathbb{Z}$, with corresponding $A$ 's satisfying

$$
-\left(h_{1} A^{\prime}\right)^{\prime}+n^{2} h_{2} A=\lambda h_{3} .
$$

Standard ODE theory shows that for fixed $n \in \mathbb{Z}$ the set of such $A$ with Neumann condition span $L^{2}[L, R]$, and so such solutions $u(t, \theta)=$ $A(t) e^{i n \theta}$ give a basis for $L^{2}\left(M_{L, R}\right)$. Hence if $f$ depends on $\theta$, then $\lambda_{1}=\lambda$ for some Neumann solution to Equation (5) with $n \neq 0$. But such a $\lambda$ equals the Rayleigh quotient $\mathcal{R}_{n}(A)$ with

$$
\mathcal{R}_{n}(A)=\frac{\int_{L}^{R}\left[h_{1}\left(A^{\prime}\right)^{2}+n^{2} A^{2} h_{2}\right] d t}{\int_{L}^{R}\left[A^{2} h_{3}\right] d t} .
$$

Ignoring the clearly non-negative $h_{1}\left(A^{\prime}\right)^{2}$ term in the numerator, we see that

$$
\mathcal{R}_{n}(A) \geq \frac{\int_{L}^{R}\left[n^{2} A^{2} h_{2}\right] d t}{\int_{L}^{R}\left[A^{2} h_{3}\right] d t} \leq n^{2} \min \left(h_{2} / h_{3}\right)=n^{2} e^{2 L} .
$$

It then follows that

$$
\lambda_{1} \geq n^{2} e^{2 L} \geq e^{2 L},
$$

which certainly exceeds $1 / 4$ when $L \geq 0$.

\section{References}

[1] N. Alon \& V. D. Milman, $\lambda_{1}$, isoperimetric inequalities for graphs, and superconcentrators, J. Combin. Theory Ser. B 38 (1985) 73-88.

[2] S. Bobkov \& M. Ledoux, Poincaré's inequalities and Talagrand's concentration phenomenon for the exponential distribution, Probab. Theory Related Fields 107 (1997) 383-400. 
[3] Robert Brooks, On the spectrum of noncompact manifolds with finite volume, Math. Z. 187 (1984) 425-432.

[4] F. R. K. Chung, V. Faber \& Thomas A. Manteuffel, An upper bound on the diameter of a graph from eigenvalues associated with its Laplacian, SIAM J. Discrete Math. 7 (1994) 443-457.

[5] F. R. K. Chung, A. Grigor'yan \& S.-T. Yau, Upper bounds for eigenvalues of the discrete and continuous Laplace operators, Adv. Math. 117 (1996) 165-178.

[6] _ Eigenvalues and diameters for manifolds and graphs, Tsing Hua lectures on geometry \& analysis (Hsinchu, 1990-1991) Internat. Press, Cambridge, MA, 1997, 79-105.

[7] F. R. K. Chung, Laplacians of graphs and Cheeger inequalities, Combinatorics Paul Erdös is eighty, Bolyai Soc. Math. Stud., 1993, 1-16.

[8] J. Dodziuk \& W. S. Kendall, Combinatorial Laplacians and isoperimetric inequality, From local times to global geometry, control and physics, 1986.

[9] Jozef Dodziuk \& Leon Karp, Spectral and function theory for combinatorial Laplacians, Geometry of Random Motion, Contemp. Math. 73 (1988) 25-40.

[10] Joel Friedman \& Jean-Pierre Tillich, Wave equations for graphs and the edge based Laplacian, to appear.

[11] A. Lubotzky, R. Phillips \& P. Sarnak, Ramanujan graphs, Combinatorica 8 (1988) 261-277.

[12] Bojan Mohar, Eigenvalues, diameter, and mean distance in graphs, Graphs Combin. 7 (1991) 53-64.

[13] Vitali D. Milman \& Gideon Schechtman, Asymptotic theory of finite-dimensional normed spaces, Springer, Berlin, 1986. With an appendix by M. Gromov.

[14] P. Sarnak, Some applications of modular forms, Cambridge Tracts in Math., Vol. 99, Cambridge Univ. Press, Cambridge, 1990.

\author{
University of British Colubmia, Canada \\ Université Paris-Sud, France
}

